\title{
Your pain, my gain: The relationship between self-report and behavioral measures of everyday sadism and COVID-19 vaccination intention
}

\author{
Heng $\mathrm{Li}^{1} \cdot \mathrm{Yu} \mathrm{CaO}{ }^{2}$
}

Accepted: 24 January 2022

@ The Author(s), under exclusive licence to Springer Science+Business Media, LLC, part of Springer Nature 2022

\begin{abstract}
Vaccination plays a crucial role in containing the spread of the COVID-19 pandemic. However, a significant fraction of the global population is reluctant to take a coronavirus vaccine. A burgeoning literature has considered mainly adaptive personality traits as antecedents of vaccine hesitancy (i.e., Big Five and HEXACO), while maladaptive personality traits (i.e., "Dark Tetrad" of personality) are often a comparatively neglected area. In this research, we examined the relationship between everyday sadism and intention to get vaccinated against COVID-19. We theorized that driven by antisocial tendencies and social indifference, individuals with higher sadism may be less willing to obtain a vaccine. Employing a bug-killing paradigm to capture everyday sadism, we tested this prediction in a Chinese sample of non-student adults $(\mathrm{N}=188)$. Support for this proposition was found in the lab task, which demonstrates that sadism was associated with more vaccine refusal spanning the self-report and behavioral domains. In addition, we showed that the sadistic behavioral choices can be predicted with self-report measure of sadistic personality. These findings highlight the important role of maladaptive personality traits in predicting vaccination attitudes and intentions.
\end{abstract}

Keywords Sadism, sadistic personality $\cdot$ Behavioral measures $\cdot$ Vaccination attitudes $\cdot$ COVID-19 $\cdot$ Vaccination hesitancy

\section{Introduction}

The new coronavirus disease (COVID-19) poses the greatest threat to public health in recent years (World Health Organization, 2020). This global health crisis has disrupted lives, created economic recession, and pushed the healthcare system to its capacity (Burki, 2020; Chakraborty \& Maity, 2020; Susskind \& Vines, 2020). Many scientists believe that successful vaccine rollouts are crucial for reaching the herd immunity threshold and for slowing the relentless march of the pandemic (Altmann et al., 2020; Ledford et al., 2020). However, a significant fraction of the global population is reluctant to take a coronavirus vaccine due to various reasons such as mistrust of healthcare providers

Heng Li

leehem168@163.com

1 College of International Studies, Southwest University, Chongqing 400715, China

2 School of Foreign Languages, Zhongnan University of Economics and Law, Wuhan, China and anti-vaccination infodemic on social media (Diseases, 2020; Palamenghi et al., 2020). Much evidence has shown that vaccine hesitancy has been one of the chief factors accounting for the failure of meeting their COVID-19 vaccination benchmarks, along with inequitable distributions and manufacturing bottlenecks (Bollyky et al., 2020; Dror et al., 2020). The reasons for COVID-19 vaccine hesitancy remain complex. In the past few decades, research on vaccination hesitancy has been focused on a rich variety of health, demographic, and psychological factors (Huynh \& Senger, 2021; Malik et al., 2020). However, the picture that has emerged from these investigations is not complete without considering the role of personality.

For more than 50 years, developments in the field of personality psychology suggest that people's sets of behavioral performance, cognitive process, and judgement and decision making vary in ways predicted by broad personality variables (Cantor, 1990; George, 1990; Mendes et al., 2019). Drawing on this literature, we suggest that personality should receive more attention in the study of vaccination attitudes and behavior for at least two reasons. One, the exploration of personality roots of vaccination intention 
could help identify ways to reduce vaccine hesitancy and its impact on public health. Two, the identification of personality traits that moderate the vaccination acceptance could refine existing theories or advance new theories, which could eventually help to advance our understanding of vaccination hesitancy.

Within multiple models of both normal-range and maladaptive personality traits, the dark triad model is perhaps one of the most used grouping, consisting of three overlapping but distinctive traits: narcissism (characterized by egotistic-admiration and self-involvement), Machiavellianism (centering on interpersonal manipulation and callousness), and psychopathy (marked by the absence of empathy and socially irresponsible behavior) (Jones \& Paulhus, 2010; Vernon et al., 2008; Paulhus \& Williams, 2002; for a comprehensive review of the dark-triad literature, see Miller et al., 2019). Recently, a fourth unique personality trait, sadism, has been added to that model, composed of the "Dark Tetrad" (Chabrol et al., 2009). In the literature, sadism is defined as the tendency to derive pleasure from a conscious victim who undergo psychological, emotional, or physical suffering (Paulhus \& Dutton, 2016).

The current research aims to explore the relationship between self-report and behavioral measures of everyday sadism and vaccination attitudes. Our study offers three important contributions to the existing literature. To begin with, the specific association between dark personalities, especially sadism, and vaccination intention has been scarcely investigated. Second, most studies on personality determinants of vaccination attitudes worked with populations from WEIRD (Western, Educated, Industrialized, Rich, and Democratic) societies which are unrepresentative of human culture more globally (Henrich et al., 2010). Finally, the vast majority of literature has exclusively investigated vaccination attitudes rather than vaccination behavior. It is possible that there is a striking association between attitudes and behavior (Cao and $\mathrm{Li}, 2021$ ). To address this issue, we examined vaccination acceptance by asking respondents to make a behavioral choice.

\section{Literature Review}

To date, a limited amount of research has demonstrated that some aspects of personality traits are correlated with vaccination attitudes (Carvalho et al., 2020). Scholars have tested two frameworks of the structure of personality traits, namely, the Five Factor Model (extraversion, agreeableness, openness, conscientiousness, and neuroticism) (Lin $\&$ Wang, 2020) and the Big Six dimension or HEXACO (honesty-humility, emotionality, extraversion, agreeableness, conscientiousness, and openness to experience) model of personality structure (Lee et al., 2017).
With respect to the Five Factor Model, an earlier study reported by Browne et al. (2015) investigated the relationship between human traits of personality openness and vaccination skepticism in a nationally representative sample of Australians. It was found that the personality trait of openness to experience was negatively related to perceptions toward vaccination. One possible reason for the inverse relation is that individuals with a higher level of openness are less likely to acquire knowledge from traditional and authority sources. Despite this research providing empirical evidence for personality roots of vaccination hesitancy, it focused exclusively on the role of openness to experience. Thus, the effects of the other five personality traits are unclear and it is an open question whether the observed relationship still holds when controlling for other personality variables.

As for the HEXACO model of personality, Lee et al. (2017) evaluated the effect of the six major dimensions of personality on confidence in the safety of childhood vaccinations in a national probability sample of New Zealand adults. They found that people who evidenced a lower level of conscientiousness and agreeableness but a higher level of openness to experience expressed more concerns about vaccine safety. Such findings provide insight into the personality factors underlying parental vaccine confidence. In a similar vein, Lin and Wang (2020) examined the relationship between Big Five personality traits and individual perceptions of vaccination in a sample of 3276 American citizens. In line with previous research findings, they found that individuals high on the traits of conscientiousness and agreeableness showed more positive attitudes toward vaccination even adjusting for other social and demographic variables. These findings suggest that personality dispositions are significantly associated with differences in individuals' vaccination attitudes and intention.

More recently, an emerging line of research has replicated and extended the findings regarding the relationship between personality traits and vaccine acceptance in the context of the COVID-19 pandemic (Hughes \& Machan, 2021). For example, Murphy et al. (2021) found that lower scores in the personality traits agreeableness and conscientiousness and higher scores in neuroticism positively predicted COVID-19 vaccine hesitancy and resistance in a large UK sample. Likewise, Tanaka et al. (2021) examined the association between 17 social personality traits (e.g., neuroticism, conscientiousness, and altruism) and the willingness of COVID-19 vaccination in a large Japanese sample $(\mathrm{N}=6232)$. The results showed that many BIG 5 personality traits like agreeableness were significant predictors of the willingness to be vaccinated. Thus, these consistent findings advance our understanding of the personality roots of individual perceptions of vaccinations. 
Despite previous research highlighting the importance of personality traits in predicting vaccination attitudes and intentions, it also has some limitations. One problem is that prior work has primarily focused on adaptive personality traits as antecedents of vaccine hesitancy by employing the most widely used multiscale personality models (i.e., Big Five and HEXACO), while maladaptive personality traits have often been neglected in the existing literature. Although little attention has been directed to understand the role of dark personalities in vaccine attitudes, a burgeoning stream of research has examined the associations between dark personality traits and safety protocols such as mask wearing and social distancing in the fight against the COVID19 pandemic (Hughes \& Machan, 2021; Li, 2021). For example, Zajenkowski et al. (2020) found that individuals who evidenced a higher level of narcissistic rivalry, Machiavellianism, and psychopathy were less likely to engage in health-promoting behavior (e.g., physical distance and hygienic behaviors) during a pandemic of a contagious respiratory disease. Likewise, in a survey involving 502 online respondents, Blagov (2020) found that some Dark traits such as psychopathy, meanness, and disinhibition were associated with a lower likelihood of health behaviors endorsement. These findings suggest that borderline personality traits are meaningful predictors of compliance with COVID-19 preventive measures, although they may be only responsible for a small percentage of the variance.

\section{Theory and Hypothesis Development}

In contrast to a relative abundance of empirical work on the association between Dark Triad of personality and safety protocols to COVID-19 transmission, there is a paucity of information available on the effect of sadism. Two lines of reasoning related to antisocial tendencies and to enjoyment of upheaval caused by the pandemic suggest that everyday sadism may be associated with a more negative intention to have a vaccination. On the one hand, sadism is characterized by strong tendencies for antisocial behavior, such as a desire to inflict harm or humiliation on others (Moor \& Anderson, 2019). For example, Chabrol et al. (2009) assessed the relative contributions of psychopathic, narcissistic, Machiavellian, and sadistic traits to juvenile delinquency in a sample of 615 high-school students. The results showed that individuals with higher sadistic propensities displayed more antisocial behavior independently of its construct overlap with the Dark Triad clusters. Applying this logic to the domain of public health, refusing to be vaccinated may expose antivaxxers themselves and other people to viruses and create barriers to achieve herd immunity. Thus, choosing no vaccine can be viewed as an expression of antisocial propensity to placing individuals' own feelings ahead of the welfare of the general population.
On the other hand, recent research suggests that high-sadism individuals, characterized by feelings of excitement to get pleasure from hurting others or watching their suffering, may perceive the pandemic differently (Lui et al., 2020). For example, Hardin et al. (2021) found that American adults who scored higher on measures of sadism experienced more positive emotions in response to the new coronavirus disease. This is possibly because those with high levels of sadism hold no concerns about social instability caused by the pandemic. Combining these two lines of reasoning, we therefore hypothesize:

Hypothesis 1. Individuals who score higher on selfreport and behavioral measures of sadism would have more negative attitudes and low intention to vaccinate since they may experience pleasure when COVID-19 caused a tsunami of suffering.

By offering a behavioral demonstration of everyday sadism, a study by Buckels et al. (2013) is informative. They used a modified coffee grinder that provided participants the chance to get ride of bugs in a rather cruel manner but, in reality, no bugs were actually killed. In addition to this bugcrunching task, there are other unsavory tasks (e.g., cleaning toilets, working in cold temperatures) that participants could choose. It was found that those who chose the bug-crunching task evidenced a higher level of everyday sadism on a self-report questionnaire measure. This pattern of results suggests that everyday sadism is an added dark personality and can be captured in a laboratory context. One point for consideration is that the sample in Buckels et al. (2013) was heavily biased towards psychology students from a WEIRD country. Although this study is a useful starting point, a cross-cultural test of the observed relationship can increase the generalizability of their findings. Thus, this leads to our second hypothesis:

Hypothesis 2. sadism scores would be linked to a greater likelihood of sadistic behavioral choices in a laboratory setting.

\section{Method}

\section{Participants}

Participants were recruited via online advertising, community informants, and flyers in central China. To restrict the opportunistic use of researcher degrees of freedom, we decided in advance to collect as many as participants over a two-week period. No participants were added after the initial statistical analysis. Inclusion criteria required participants to be native speakers of Chinese, of Chinese nationality and of 
Han ethnicity, and aged 18 years or over. At the screening stage, no prospective participants reported that they suffered from comorbidity of mood, anxiety, and substance use disorders and took psychiatric medications according to their self-report. All procedures performed in studies involving human participants were in accordance with the ethical standards of the institutional and/or national research committee and with the 1964 Helsinki declaration and its later amendments or comparable ethical standards.

A total of 217 potential participants registered online to take part in the study in exchange for 20 yuan RMB. However, twenty-five registrants did not show up for the study. Data from another four participants were excluded from the analysis because the critical data regarding their choices were not recorded. Thus, the final sample consisted of 188 participants (99 women and 89 men), ranging in age from 18 to 58 years $(M=35.7, S D=11.4)$. All participants had not received a vaccine against COVID-19 when the study took place. The post hoc analysis showed that the observed power of the study was 0.76 .

\section{Materials and Procedure}

Upon arrival at the lab, participants were told that researchers were interested in their job preference. First, participants completed the Assessment of Sadistic Personality, a 10-item measure of an individual's everyday form of sadism (O'Meara et al., 2011). They were asked to indicate their agreement on a 5-point response scale, ranging from 1 (strongly disagree) to 5 (strongly agree). Example items include "Watching people get into fights excites me" and "I think about hurting people who irritate me" (Croabach's alpha was 0.87). Next, participants were asked to respond to the 16-item Disgust Propensity and Sensitivity ScaleRevised (van Overveld et al., 2006), a common measure of choice for assessing the disgust sensitivity (Croabach's alpha was 0.81). Sample items include "Disgusting things make my stomach turn" and "When I experience disgust, it is an intense feeling". Previous published studies have shown that the Chinese version of these two questionnaires demonstrates sufficient psychometric properties in Chinese populations (Liu et al., 2019; Yang et al., 2018).

This study used a quasi-experimental design to assess the association between everyday sadism and vaccination attitudes and the congruence between overt sadistic behavior and its personality predictors. Closely following the bugkilling paradigm which was used to capture the behavioral choice of everyday sadism in Buckels et al. (2013), we asked participants to choose one of the four unpleasant job options shown below. The order of options was randomized and counterbalanced across subjects.

1. Exterminator: eliminating the unwanted pests.
2. Exterminator's assistant: helping the experimenter get rid of bugs.

3. Sanitation worker: cleaning toilets, emptying pits and septic tanks.

4. A worker in cold temperatures: enduring the effects of ice-water immersion.

If participants chose to take the position as an exterminator, we showed them a "bug-crunching machine", but it is actually a modified coffee grinder which produced a distinct grinding noise in order to increase the believability of the cover story. Each of three cups which contained a sow bug was placed close to the machine. The bugs' names-Liming, Lihua, and Liwen-were printed on the containers. The participant's job was to force the insects into the machine and crush them into small pieces. The experimenter stood far from the participants and pretended to arrange the files. To comply with animal welfare standards, we modified the design of the machine, unbeknownst to participants, to prevent the insects from getting into the grinding blades. Therefore, participants were led to believe that the bugs were being grinded up, but none of these insects were actually damaged in the experiment. When participants began to kill the bugs, the research assistant recorded the number of bugs participants killed $(M=1.25$, $S D=0.95$ )

For participants choosing the option as the exterminator's assistant, they did not need to grind up the bug but simply passed the containers to the research assistant. Participants who chose the role of sanitation worker were presented bathroom-cleaning tools for toilet washing, while those who chose to work in a cold environment were informed that the temperature calibration equipment was placed in another room. Once participants completed the personality questionnaires and these challenging jobs, the research assistant said:

"Thank you for taking the time and effort in doing the task. Here is 20 yuan for your participation. Ahh, actually I forgot to tell you that our university has some cooperation with local hospitals and thus we received some slots for a coronavirus (COVID-19) vaccination at a reliable community pharmacy. Now we decided to distribute them to our participants. Would you like us to book a vaccination appointment for you?"

After participants made a choice (yes/no), they were eventually debriefed about the true nature of the study and that the vaccination appointment opportunities did not exist. Though participants were not getting vaccinated in the study due to ethical restrictions, they indeed made a behavior choice under this circumstance. 


\section{Results and Discussion}

Debriefing responses indicated that no participants issued suspicion or awareness about the veracity of the cover story. This is possibly because it is not uncommon to receive free gifts when taking part in a study. Table 1 illustrates the descriptive statistics of sadism scores across different groups. $28.2 \%$ chose to eliminate the unwanted pests, and $29.3 \%$ chose to help treat bugs, $16.5 \%$ chose the pain-tolerance task, and $26.1 \%$ chose the sanitation task. There were no significant differences across the task choices in terms of gender, age, and disgust sensitivity, $p s>0.25$.

As we expected, participants who chose to be an exterminator displayed the highest level of sadism. Following Buckels et al. (2013), we used sadism scores to predict the sadistic behavioral choices (on a continuum with pest control as the most sadistic option, followed by assisting with pest control, and then sanitation and pain tolerance grouped as the least-sadistic choices) in an ordered logistic regression when accounting for age, gender, and disgust sensitivity. Consistent with Hypothesis 2, we found that everyday sadism was a significant and positive predictor of the task choices, Wald $(\mathrm{df}=1)=10.32, p=0.001$, odds ratio $=-0.76$ (95\% confidence interval $[\mathrm{CI}]=-1.251,-0.299)$.

We then conducted a logistic regression analysis to predict vaccination behavior $(0=$ vaccine refusal, $1=$ vaccine acceptance) by using sadism scores. Consistent with Hypothesis 1, we found that higher sadism scores were associated with greater odds of declining vaccination opportunities, Wald $(\mathrm{df}=1)=5.99, p=0.014$, odds ratio $=0.44(95 \%$ confidence interval $[\mathrm{CI}]=0.225,0.848$ ). Finally, we used the sadistic behavioral choices to predict vaccination behavior in the same model. Again, we found that sadistic behavior was significantly positively predictive of vaccine refusal, Wald $(\mathrm{df}=1)=15.38, p<0.001$, odds ratio $=1.72(95 \%$ confidence interval $[\mathrm{CI}]=1.313,2.264)($ Table 2$)$.

\section{General Discussion}

Based on findings that everyday sadism is associated with antisocial tendencies and social indifference (Moor \& Anderson, 2019; Paulhus \& Dutton, 2016), we propose that sadistic personality traits and behavior can significantly predict vaccination attitudes. To test our theoretical perspective, we assessed sadism subjectively with self-report and objectively with behavioral choices in a Chinese sample of non-student adults $(\mathrm{N}=188)$. In line with our prediction, we found that participants who exhibited a higher level of everyday sadism were less likely to obtain a vaccination against COVID-19 in a behavioral context. These results highlighted the important role of everyday sadism in individuals' perceptions of vaccination.

\section{Theoretical Contributions}

This research provides clear theoretical contributions. To begin with, a limited number of studies offered preliminary evidence for the behavioral conformation of everyday sadism (Buckels et al., 2013). However, most of them were based on WEIRD populations, which indicates a restricted range of generalizability of these research findings. Accumulating research findings suggest that the structure of personality and its effect on human behavior may vary across cultures (Paunonen et al., 1992). For instance, Kitayama and Park
Table 1 Means and standard deviations for sadism traits across different groups
Table 2 Results of regression models of sadism scores, sadistic behavior, and task choices

\begin{tabular}{|c|c|c|c|c|c|c|}
\hline \multirow[t]{2}{*}{ Group } & \multicolumn{2}{|c|}{ Across groups } & \multicolumn{2}{|c|}{ Vaccine acceptance } & \multicolumn{2}{|c|}{ Vaccine refusal } \\
\hline & $M$ & $S D$ & $M$ & $S D$ & $M$ & $S D$ \\
\hline $\begin{array}{l}\text { Exterminator } \\
\text { (Acceptance: } 7 \text {, refusal: 46) }\end{array}$ & 1.80 & 0.74 & 1.61 & 0.66 & 1.83 & 0.76 \\
\hline $\begin{array}{l}\text { Exterminator's assistant } \\
\text { (Acceptance: } 24 \text {, refusal: } 31 \text { ) }\end{array}$ & 1.53 & 0.57 & 1.40 & 0.24 & 1.64 & 0.71 \\
\hline $\begin{array}{l}\text { Sanitation worker } \\
\text { (Acceptance: } 15 \text {, refusal: } 16 \text { ) }\end{array}$ & 1.44 & 0.54 & 1.40 & 0.47 & 1.47 & 0.62 \\
\hline $\begin{array}{l}\text { Worker in cold environment } \\
\text { (Acceptance: } 26 \text {, refusal: } 23 \text { ) }\end{array}$ & 1.43 & 0.49 & 1.41 & 0.49 & 1.46 & 0.23 \\
\hline
\end{tabular}

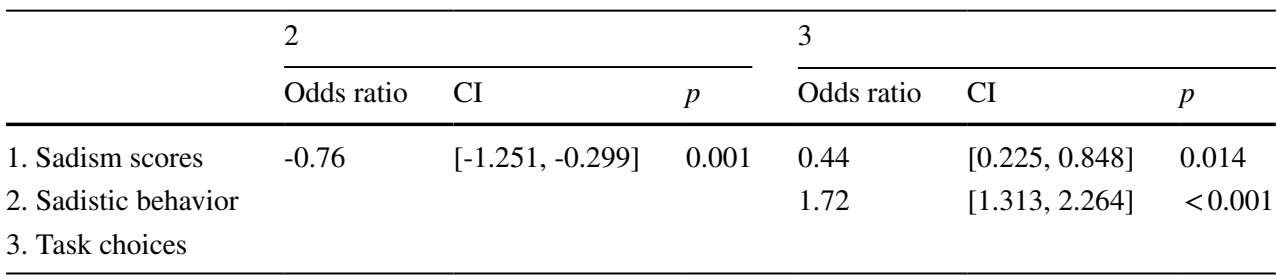


(2021) found that despite the strong relationship between conscientiousness and better health in Western societies, conscientiousness was associated with higher biological health risk (BHR) in Eastern Asian societies. This is possibly because conscientiousness personality traits were associated with more compliance with social responsibility, which in turn predicted higher BHR. Our analysis of Chinese data, therefore, was the first test of whether there is a congruence between overt sadistic behavior and its personality predictors in Chinese participants, a clear example of non-WEIRD populations. We found that sadists showed the greatest preference for taking the job of eliminating the unwanted pests. This pattern of results suggests that sadism played a unique role in predicting this concrete behavior in everyday life (or at least in a laboratory setting).

Second, some prior work on the relationship between dark personality traits and public health recommendations related to COVID-19 proved to be inconclusive. For instance, Zajenkowski et al. (2020) found that dark personalities were meaningful predictors of breaching coronavirus restrictions. In contrast, Hardin et al. (2021) found no evidence for any direct association between dark personalities and self-reports of social distancing compliance. This discrepancy might be related to time points of the experiments. Since personality can only explain a small percentage of the variance, other factors such as health risk perception may override a personality factor to shape people's adherence to safety protocols due to the severity of COVID-19 (Caserotti et al., 2021; Commodari et al., 2020; Qiao et al., 2021). In addition, Hardin et al. (2021) found that sadism was significantly positively predictive of hygiene behavior despite being difficult to interpret according to theories of personality and individual differences. However, the role of sadism in vaccination attitudes has not been tested in previous research. Thus, our study represents the first attempt to investigate the relationship between everyday sadism and vaccination-related behavior.

Our work suggests that both self-report and behavioral measures of everyday sadism are negatively associated with people's behavioral choice regarding vaccination against the new coronavirus disease. This is in line with previous findings that individuals who are higher in sadism may perceive the threat of the pandemic differently (Hardin et al., 2021; Hughes \& Machan, 2021). Specially, individuals possessing sadistic personalities may show indifference to other humans and lack of compassion toward those who suffer physical, emotional, and psychological damages caused by the pandemic. Thus, these findings suggest that the pleasure of eliminating pests may extend to the enjoyment of social instabilities such as mass infection caused by vaccine refusal. Though a vast majority of existing studies tend to construe sadism as a personality disorder such as sadomasochism in hardened criminals (Mokros et al., 2011) and in sexual offenders (Marshall \& Kennedy, 2003), our results advance the literature by showing the robust relationship between everyday sadism and deviations from health guidelines. Yet, since only very few studies tested the role of sadism in preventive measures surrounding COVID-19, it is unclear whether sadism is only associated with vaccination hesitancy or with more health protective behaviors in general.

\section{Practical Implications}

In addition to theoretical contributions, some practical implications arise from the current study. Given that our findings suggest that individuals with high sadism may hold more negative attitudes toward vaccination, governments and organizations during a vaccination campaign must pay special attention to those people since vaccine hesitancy can have higher healthcare costs. As such, interventions and practices, such as raising effective awareness about the importance of vaccination for public health can be implemented to address vaccine hesitancy in individuals with high dispositional sadism. For example, organizations and institutions must ensure that they put in place mechanisms to monitor and detect sadistic traits among staff and develop internal controls capable of mitigating negative effects of everyday sadism. Internal controls should focus on ethical cultures and compliance programs during COVID-19 and create a positive workplace culture capable of inhibiting abusive behaviors that may detrimentally influence community safety and health (Góis et al., 2020).

\section{Limitations and Future Directions}

Although this study represents a useful starting point for a more complete understanding of the role of everyday sadism in vaccination attitudes, several limitations of the research need to be noted. First, our evidence is restricted to normal populations in China. Therefore, it is recommended that future research test the relationship studies here in other parts of the world and in clinical populations to determine whether these research findings can be replicated and generalized. Second, though we recruited participants from a broad cross-section of society, the convenience sample was not quota matched to the Chinese population on many demographic variables such as age, gender, occupations, and educational attainment. Third, as the cross-sectional design of our study is observational in nature, the correlational data precludes the assessment of causality. A longitudinal study would be valuable to test the causal relationship. Fourth, practitioners should be cautious when interpreting results of behavioral choices 
because participants did not actually get vaccinated during the experiment. Fifth, the current study focused exclusively on sadistic traits; future research can be conducted to explore the effect of its interaction with other dark personalities on vaccination attitudes. Finally, the small percentage of variance explained by sadism scores alone indicates that other factors such as sociodemographic characteristics of participants (e.g., area of residence, education level, and socioeconomic status) are likely important. Future research can integrate these other sources of variance to better understand psychological roots of vaccination behavior.

\section{Conclusion}

In a world where the new coronavirus disease has infected and killed millions of people, it is critical that people get vaccinated to build protection. Meanwhile, research should keep pace with these developments and seek to understand factors affecting attitudes towards COVID-19 vaccination. In this vein, our study demonstrates that everyday sadism is a significant and unique predictor of vaccination behavior. We outlined an approach to the study of sadism that overcomes some of the methodological problems arising from other approaches and better reflects sadistic behavior in a laboratory setting. The current study advances the literature on the construct validity of everyday sadism and on the personality antecedents of vaccine hesitancy. Given interventions have demonstrated that sadistic behavior can be modified (Beech \& Harkins, 2012), the potential role of psychosocial interventions in clinical populations to encourage vaccination should be explored in future research.

Funding This work was supported by Social Science Foundation of Chongqing Municipality of China (2019BS020) and the Fundamental Research Funds for the Central Universities, Southwest University (SWU1909753)

Data Availability Statement The data that support the findings of this study are available from the corresponding author upon reasonable request.

\section{Declarations}

Ethical Approval All procedures performed in studies involving human participants were in accordance with the ethical standards of the institutional (Zhongnan University of Economics and Law)and/or national research committee and with the 1964 Helsinki declaration and its later amendments or comparable ethical standards.

Informed Consent Informed consent was obtained from all participants.
Conflict of Interest The authors declared no conflict of interest.

\section{References}

Altmann, D. M., Douek, D. C., \& Boyton, R. J. (2020). What policy makers need to know about COVID-19 protective immunity. The Lancet, 395(10236), 1527-1529.

Beech, A. R., \& Harkins, L. (2012). DSM-IV paraphilia: Descriptions, demographics and treatment interventions. Aggression and Violent Behavior, 17(6), 527-539.

Blagov, P. S. (2020). Adaptive and dark personality traits in the COVID-19 pandemic: Predicting health-behavior endorsement and the appeal of public health messages. PsyArXiv Preprints. https://doi.org/10.31234/osf.io/chgkn

Bollyky, T. J., Gostin, L. O., \& Hamburg, M. A. (2020). The equitable distribution of COVID-19 therapeutics and vaccines. JAMA, 323(24), 2462-2463.

Browne, M., Thomson, P., Rockloff, M. J., \& Pennycook, G. (2015). Going against the herd: psychological and cultural factors underlying the 'vaccination confidence gap.' PLoS One, 10(9), e0132562.

Buckels, E. E., Jones, D. N., \& Paulhus, D. L. (2013). Behavioral confirmation of everyday sadism. Psychological Science, 24(11), 2201-2209.

Burki, T. (2020). COVID-19 in latin america. The Lancet Infectious Diseases, 20(5), 547-548.

Cantor, N. (1990). From thought to behavior:" Having" and" doing" in the study of personality and cognition. American Psychologist, 45(6), 735-750

Cao, Y., \& Li, H. (2021). Towards controlling of a pandemic: How self-control ability influences willingness to take the COVID-19 vaccine. Personality and Individual Differences, 188, 111447.

Carvalho, L. D. F., Pianowski, G., \& Gonçalves, A. P. (2020). Personality differences and COVID-19: Are extroversion and conscientiousness personality traits associated with engagement with containment measures? Trends in Psychiatry and Psychotherapy, 42(2), 179-184

Caserotti, M., Girardi, P., Rubaltelli, E., Tasso, A., Lotto, L., \& Gavaruzzi, T. (2021). Associations of COVID-19 risk perception with vaccine hesitancy over time for Italian residents. Social Science $\&$ Medicine, 272, 113688.

Chabrol, H., Van Leeuwen, N., \& RodgersS'ejourn'e, R. N. (2009). Contributions of psychopathic, narcissistic, Machiavellian, and sadistic personality traits to juvenile delinquency. Personality and Individual Differences, 47(1), 734-739.

Chakraborty, I., \& Maity, P. (2020). COVID-19 outbreak: Migration, effects on society, global environment and prevention. Science of the Total Environment, 728, 138882.

Commodari, E., La Rosa, V. L., \& Coniglio, M. A. (2020). Health risk perceptions in the era of the new coronavirus: Are the Italian people ready for a novel virus? A cross-sectional study on perceived personal and comparative susceptibility for infectious diseases. Public Health, 187, 8-14.

Diseases, T. L. I. (2020). The COVID-19 infodemic. The Lancet. Infectious Diseases, 20(8), 875.

Dror, A. A., Eisenbach, N., Taiber, S., Morozov, N. G., Mizrachi, M., Zigron, A., ... \& Sela, E. (2020). Vaccine hesitancy: the next challenge in the fight against COVID-19. European Journal of Epidemiology, 35(8), 775-779.

George, J. M. (1990). Personality, affect, and behavior in groups. Journal of Applied Psychology, 75(2), 107-116.

Góis, A. D., Lima, G. A. S. F. D., \& De Luca, M. M. M. (2020). Everyday sadism in the business area. RAUSP Management Journal, $55,393-408$. 
Hardin, B. S., Smith, C. V., \& Jordan, L. N. (2021). Is the COVID-19 pandemic even darker for some? Examining dark personality and affective, cognitive, and behavioral responses to the COVID-19 pandemic. Personality and Individual Differences, 171, 110504.

Henrich, J., Heine, S. J., \& Norenzayan, A. (2010). Most people are not WEIRD. Nature, 466(7302), 29-29.

Hughes, S., \& Machan, L. (2021). It's a conspiracy: Covid-19 conspiracies link to psychopathy, Machiavellianism and collective narcissism. Personality and Individual Differences, 171, 110559.

Huynh, H. P., \& Senger, A. R. (2021). A little shot of humility: Intellectual humility predicts vaccination attitudes and intention to vaccinate against COVID-19. Journal of Applied Social Psychology, 51(4), 449-460.

Jones, D. N., \& Paulhus, D. L. (2010). Different provocations trigger aggression in narcissists and psychopaths. Social Psychological \& Personality Science, 1, 12-18.

Kitayama, S., \& Park, J. (2021). Is conscientiousness always associated with better health? A US-Japan cross-cultural examination of biological health risk. Personality and Social Psychology Bulletin, 47(3), 486-498.

Ledford, H., Cyranoski, D., \& Van Noorden, R. (2020). The UK has approved a COVID vaccine-here's what scientists now want to know. Nature, 588(7837), 205-206.

Lee, C. H., Duck, I. M., \& Sibley, C. G. (2017). Personality and demographic correlates of New Zealanders' confidence in the safety of childhood vaccinations. Vaccine, 35(45), 6089-6095.

Li, H. (2021). Follow or not follow?: The relationship between psychological entitlement and compliance with preventive measures to the COVID-19. Personality and Individual Differences, 174, 110678.

Lin, F. Y., \& Wang, C. H. (2020). Personality and individual attitudes toward vaccination: A nationally representative survey in the United States. BMC Public Health, 20(1), 1-8.

Liu, X., Li, J., Turel, O., Chen, R., \& He, Q. (2019). Food-specific inhibitory control mediates the effect of disgust sensitivity on body mass index. Frontiers in Psychology, 10, 2391.

Lui, L. F., Sassenrath, C., \& Pfattheicher, S. (2020). When is your pain my gain? The use of perspective taking by everyday sadists. Personality and Individual Differences, 167, 110213.

Malik, A. A., McFadden, S. M., Elharake, J., \& Omer, S. B. (2020). Determinants of COVID-19 vaccine acceptance in the US. EClinicalMedicine, 26, 100495.

Marshall, W. L., \& Kennedy, P. (2003). Sexual sadism in sexual offenders: An elusive diagnosis. Aggression and Violent Behavior, 8(1), $1-22$.

Mendes, F. F., Mendes, E., \& Salleh, N. (2019). The relationship between personality and decision-making: A Systematic literature review. Information and Software Technology, 111, 50-71.

Miller, J. D., Vize, C., Crowe, M. L., \& Lynam, D. R. (2019). A critical appraisal of the dark-triad literature and suggestions for moving forward. Current Directions in Psychological Science, 28(4), $353-360$.

Mokros, A., Osterheider, M., Hucker, S. J., \& Nitschke, J. (2011). Psychopathy and sexual sadism. Law and Human Behavior, 35, 188-199.

Moor, L., \& Anderson, J. R. (2019). A systematic literature review of the relationship between dark personality traits and antisocial online behaviours. Personality and Individual Differences, 144, $40-55$.
Murphy, J., Vallières, F., Bentall, R. P., Shevlin, M., McBride, O., Hartman, T. K., ... \& Hyland, P. (2021). Psychological characteristics associated with COVID-19 vaccine hesitancy and resistance in Ireland and the United Kingdom. Nature Communications, 12(1), $1-15$.

O'Meara, A., Davies, J., \& Hammond, S. (2011). The psychometric properties and utility of the Short Sadistic Impulse Scale (SSIS). Psychological Assessment, 23(2), 523-531.

Palamenghi, L., Barello, S., Boccia, S., \& Graffigna, G. (2020). Mistrust in biomedical research and vaccine hesitancy: The forefront challenge in the battle against COVID-19 in Italy. European Journal of Epidemiology, 35(8), 785-788.

Paulhus, D. L., \& Dutton, D. G. (2016). Everyday sadism. In V. Zeigler-Hill \& D. K. Marcus (Eds.), The dark side of personality: Science and practice in social, personality, and clinical psychology (pp. 109-120). American Psychological Association.

Paulhus, D. L., \& Williams, K. M. (2002). The dark triad of personality: Narcissism, Machiavellianism, and psychopathy. Journal of Research in Personality, 36(6), 556-563.

Paunonen, S. V., Jackson, D. N., Trzebinski, J., \& Forsterling, F. (1992). Personality structure across cultures: A multimethod evaluation. Journal of Personality and Social Psychology, 62(3), $447-456$.

Qiao, S., Tam, C. C., \& Li, X. (2021). Risk exposures, risk perceptions, negative attitudes toward general vaccination, and COVID19 vaccine acceptance among college students in south Carolina. American Journal of Health Promotion. https://doi.org/10.1177/ 08901171211028407

Susskind, D., \& Vines, D. (2020). The economics of the COVID-19 pandemic: an assessment. Oxford Review of Economic Policy, 36(Supplement_1), S1-S13.

Tanaka, T., Nihonsugi, T., Ohtake, F., \& Haruno, M. (2021). Age-and gender-dependent differences in attitudes towards COVID-19 vaccination and underlying psychological processes. medRxiv. https:// doi.org/10.1101/2021.05.28.21257954

van Overveld, M., de Jong, P. J., Peters, M. L., Cavanagh, K., \& Davey, G. C. L. (2006). Disgust propensity and disgust sensitivity: Separate constructs that are differentially related to specific fears. Personality and Individual Differences, 41, 1241-1252.

Vernon, P. A., Villani, V. C., Vickers, L. C., \& Harris, J. A. (2008). A behavioral genetic investigation of the Dark Triad and the Big 5 . Personality and Individual Differences, 44, 445-452.

World Health Organization. (2020). Coronavirus disease (COVID19) advice for the public. Retrieved from https://www.who.int/ emergencies/diseases/novel-coronavirus-2019/advice-for-public. Accessed 6 Dec 2021.

Yang, Z., Yin, J., Cheng, Y., Liu, L., \& Zhang, F. (2018). The factor structure of sadistic personality and its relation with impulsive personality. Applied Psychology, 24(3), 203-212.

Zajenkowski, M., Jonason, P. K., Leniarska, M., \& Kozakiewicz, Z. (2020). Who complies with the restrictions to reduce the spread of COVID-19?: Personality and perceptions of the COVID-19 situation. Personality and Individual Differences, 166, 110199.

Publisher's Note Springer Nature remains neutral with regard to jurisdictional claims in published maps and institutional affiliations. 